\title{
THE NUMERICAL SOLUTION OF HELMHOLTZ EQUATION VIA MULTIVARIATE PADÉ APPROXIMATION
}

\author{
Muhammed Yiğider ${ }^{1}$, Mesut Karabacak ${ }^{2}$ \\ ${ }^{l}$ Erzurum Technical University Faculty of Science, Department of Mathematics, Erzurum /Turkey \\ ${ }^{2}$ Atatürk University Faculty of Science, Department of Mathematics, Erzurum /Turkey
}

\begin{abstract}
In this study, we consider the numerical solution of the Helmholtz equation, arising from numerous physical phenomena and engineering applications including energy systems. We make use of a Padé Approximation method for the solution of the Helmholtz equation. Firstly, Helmholtz partial differential equation had been converted to power series by two-dimensional differential transformation, then the numerical solution of the equation was put into Pade series form for accelerating convergence and decreasing computational time. Hereby, we obtained numerical solution of Helmholtz type partial differential equation.
\end{abstract}

Key Words: Helmholtz equation, Two-dimensional differential transformation, Multivariate Padé approximation, power series

\section{INTRODUCTION}

Consider Helmholtz equation on a given region $R$ in the $x y$ - plane in the following form.

$$
\nabla^{2} u+f(x, y) u=g(x, y)
$$

where $u(x, y)$ is known on the boundary of $R$. Suppose that a function $u=u(x, y)$ is the solution to the Equation (1). The boundary and initial conditions could be given by the following functions

$u(0, y)=\Psi_{1}(y), \quad u_{x}(0, y)=\Psi_{2}(y)$.

$u(x, 0)=\Psi_{3}(x), \quad u_{y}(x, 0)=\Psi_{4}(x)$.

where $\Psi_{1}(y), \Psi_{2}(y), \Psi_{3}(x), \Psi_{4}(x)$ are given functions [1].

These equations appear in a variety of physical topics and engineering applications, such as, electromagnetic waves, elastic waves in solids, bars, membranes, acoustic radiation, water wave propagation, heat conduction, nuclear energy reactors and oscillators. Also, in modelling of semiconductor device, Helmholtz equation arises frequently as an intermediate step in the solution of the non-linear Poisson problem. To solve these problems various numerical methods have been shown which include finite difference, finite element and boundary integral methods (BIM). Using these conventional methods, it has been found that fine grids and a large number of elements must be employed to get satisfactory accuracy. This requires large number of computer core and more computational time especially for the iteration scheme of the non-linear Poisson problem $[2,3,4,5]$.

Then, El-Sayed and Kaya [6] have given a general way to construct exact and series solutions to Helmholtz equations by

employing the Adomian decomposition method. Differential transformation was introduced first by Zhou [7]. In order to solve these type of equations numerically, we use multivariate Padé approximation. Thus we obtained numerical solution of Helmholtz type differential equations. The Padé approximation method used to accelerate the convergence of the power series solution for reducing computational time.

\section{TWO-DIMENSIONAL DIFFERENTIAL TRANSFORMATION}

The two-dimensional differential transform is defined as follows $[8,9,10]$,

$$
W(k, h)=\frac{1}{k ! h !}\left[\frac{\partial^{k+h} w(x, y)}{\partial x^{k} \partial y^{h}}\right]_{0,0}
$$

In this basic definition $W(k, h)$ is the transformed function and $w(x, y)$ is the original function. The transformation is named $T$ - function while upper case and lower case letters express the transformed and original functions respectively. $W(k, h)$ is the differential inverse transform defined as 


$$
w(x, y)=\sum_{k=0}^{\infty} \sum_{h=0}^{\infty} W(k, h) x^{k} y^{h}
$$

and by using Equations (4) and (5) we can rewrite it such that

$$
w(x, y)=\sum_{k=0}^{\infty} \sum_{h=0}^{\infty} \frac{1}{k ! h !}\left[\frac{\partial^{k+h} w(x, y)}{\partial x^{k} \partial y^{h}}\right]_{0,0} x^{k} y^{h}
$$

Table 1: The basic properties of one-dimensional differential transform method

\begin{tabular}{|l|l|}
\hline Original Function & Transformed Function \\
\hline$u(x, y)=g(x, y) \pm h(x, y)$ & $U(k, h)=G(k, h) \pm H(k, h)$ \\
\hline$u(x, y)=\lambda g(x, y)$ & $U(k, h)=\lambda G(k, h)$ \\
\hline$u(x, y)=\partial g(x, y) / \partial x$ & $U(k, h)=(k+1) G(k+1, h)$ \\
\hline$u(x, y)=\partial g(x, y) / \partial y$ & $U(k, h)=(h+1) G(k, h+1)$ \\
\hline$u(x, y)=x^{m} y^{n}$ & $U(k, h)=\delta(k-m, h-n)=\left\{\begin{array}{lc}1, \\
0,\end{array} \quad \begin{array}{c}\text { otherwise } \\
0\end{array}\right.$ \\
\hline$u(x, y)=\partial^{r+s} g(x, y) / \partial x^{r} \partial y^{s}$ & $U(k, h)=(k+1) \ldots(k+r)(h+1) \ldots(h+s) G(k+r, h+s)$ \\
\hline$u(x, y)=g(x, y) h(x, y)$ & $U(k, h)=\sum_{r=0}^{k} \sum_{s=0}^{h} G(r, h-s) H(k-r, s)$ \\
\hline
\end{tabular}

\section{MULTIVARIATE PADÉ APPROXIMANTS}

As known, the bivariate function $f(x, y)$ which has Taylor series expansion can be written as

$$
f(x, y)=\sum_{i, j=0}^{\infty} c_{i j} x^{i} y^{j}
$$

with around the origin [11]. The solution of univariate Padé approximation problem for

$$
f(x)=\sum_{i=0}^{\infty} c_{i} x^{i}
$$

is defined by

$$
p(x)=\left|\begin{array}{cccc}
\sum_{i=0}^{m} c_{i} x^{i} & x \sum_{i=0}^{m-1} c_{i} x^{i} & \cdots & x^{n} \sum_{i=0}^{m-n} c_{i} x^{i} \\
c_{m+1} & c_{m} & \cdots & c_{m+1-n} \\
\vdots & \vdots & \ddots & \vdots \\
c_{m+n} & c_{m+n-1} & \cdots & c_{m}
\end{array}\right|
$$

and 


$$
q(x)=\left|\begin{array}{cccc}
1 & x & \cdots & x^{n} \\
c_{m+1} & c_{m} & \cdots & c_{m+1-n} \\
\vdots & \vdots & \ddots & \vdots \\
c_{m+n} & c_{m+n-1} & \cdots & c_{m}
\end{array}\right|
$$

Now, We multiply $j$ th row in $p(x)$ and $q(x)$ by $x^{j+m-1}(j=2, \ldots, n+1)$ and then we divide $j$ th column in $p(x)$ and $q(x)$ by $x^{j-1}(j=2, \ldots, n+1)$.These results are a multiplication of numerator and denominator by $x^{m n}$. In doing so, we obtain

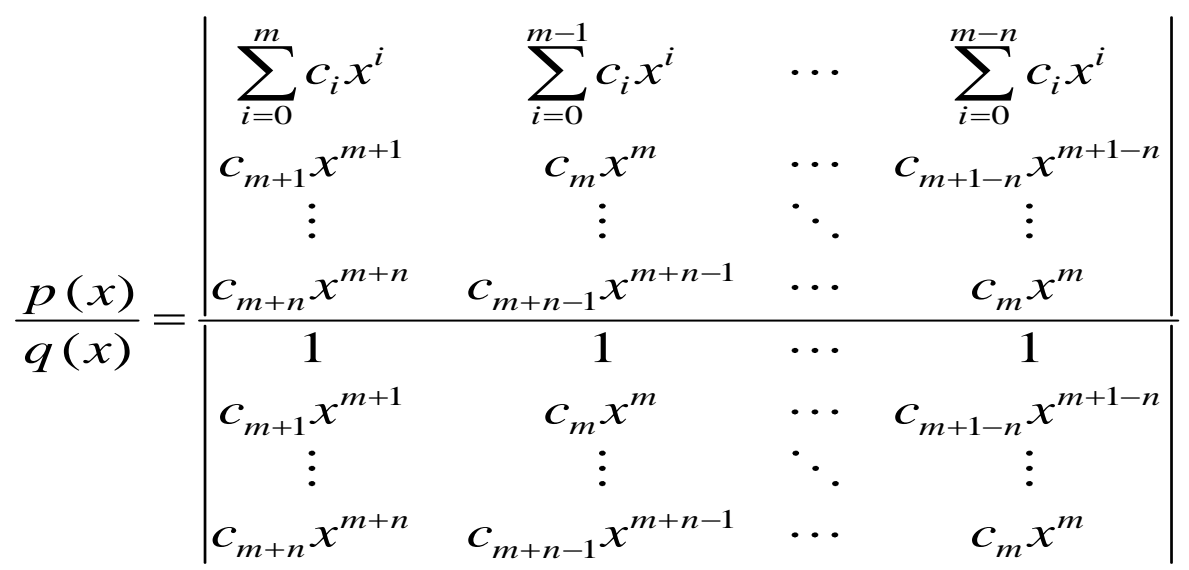

If $\left(D=\operatorname{det} D_{m, n} \neq 0\right)$

This rate of determinants can be easily obtained for a bivariate function $f(x, y)$. The sum of $\sum_{i=0}^{k} c_{i} x^{i}$ can be changed with the $k$ th partial sum of the Taylor series expansion of $f(x, y)$ and the expression $c_{k} x^{k}$ with an expression that contains all the terms of degree $k$ in $f(x, y)$. Here it is said that a bivariate term $c_{i j} x^{i} y^{j}$ is of degree $i+j$.

If we define

$$
p(x, y)=\left|\begin{array}{cccc}
\sum_{i+j=0}^{m} c_{i j} x^{i} y^{j} & \sum_{i+j=0}^{m-1} c_{i j} x^{i} y^{j} & \ldots & \sum_{i+j=0}^{m-n} c_{i j} x^{i} y^{j} \\
\sum_{i+j=m+1} c_{i j} x^{i} y^{j} & \sum_{i+j=m} c_{i j} x^{i} y^{j} & \ldots & \sum_{i+j=m+1-n} c_{i j} x^{i} y^{j} \\
\vdots & \vdots & \ddots & \vdots \\
\sum_{i+j=m+n} c_{i j} x^{i} y^{j} & \sum_{i+j=m+n-1}^{m} c_{i j} x^{i} y^{j} & \ldots & \sum_{i+j=m}^{m} c_{i j} x^{i} y^{j}
\end{array}\right|
$$

and 


$$
q(x, y)=\left|\begin{array}{cccc}
1 & 1 & \cdots & 1 \\
\sum_{i+j=m+1} c_{i j} x^{i} y^{j} & \sum_{i+j=m} c_{i j} x^{i} y^{j} & \cdots & \sum_{i+j=m+1-n} c_{i j} x^{i} y^{j} \\
\vdots & \vdots & \ddots & \vdots \\
\sum_{i+j=m+n} c_{i j} x^{i} y^{j} & \sum_{i+j=m+n-1}^{m} c_{i j} x^{i} y^{j} & \ldots & \sum_{i+j=m}^{m} c_{i j} x^{i} y^{j}
\end{array}\right|
$$

Then we can see easily that $p(x, y)$ and $q(x, y)$ are of the form

$$
\begin{aligned}
& p(x, y)=\sum_{i+j=m n}^{m n+m} a_{i j} x^{i} y^{j} \\
& q(x, y)=\sum_{i+j=m n}^{m n+n} b_{i j} x^{i} y^{j}
\end{aligned}
$$

We know that $p(x, y)$ and $q(x, y)$ are named Padé equations $[10,11,12]$. So the multivariate Padé approximant of order $(m, n)$ for $f(x, y)$ is defined as

$$
r_{m, n}(x, y)=\frac{p(x, y)}{q(x, y)}
$$

\section{NUMERICAL EXAMPLES}

Example 1: Consider the following Helmholtz equation in [13]

$$
\frac{\partial^{2} u(x, y)}{\partial x^{2}}+\frac{\partial^{2} u(x, y)}{\partial y^{2}}-u(x, y)=0
$$

with initial conditions

$$
u(0, y)=y, \quad u_{x}(0, y)=y+\cosh y
$$

The exact solution for this equation is

$$
u(x, y)=x \cosh y+y e^{x}
$$

By using the fundamental operations of two- dimensional differential transform method in Table 1, we obtained the following recurrence relation

$$
(k+1)(k+2) U(k+2, h)+(h+1)(h+2) U(k, h+2)-U(k, h)=0
$$

By applying the differential transform to initial conditions (17), we have

$$
\begin{gathered}
U(0, h)=\delta(h-1)= \begin{cases}1 & h=1, \\
0 & h \neq 1 .\end{cases} \\
U(1, h)=\delta(h-1)+T, \quad \delta(h-1)=\left\{\begin{array}{ll}
1 & h=1, \\
0 & h \neq 1 .
\end{array}, \quad T= \begin{cases}0 & h \text { is odd }, \\
\frac{1}{h !} & \text { h is even } .\end{cases} \right.
\end{gathered}
$$


So we have;

$$
U(1, h)= \begin{cases}\frac{1}{h !} & h \text { is even } \\ 1 & h=1 \\ 0 & \text { otherwise }\end{cases}
$$

For each $k, h$ substituting Equation (19) into Equation (22) and using recursive method, the values of $U(k, h)$ can be evaluated as follows:

$$
\begin{array}{ll}
U(0, h)=0 & h=0,2,3,4 \ldots \\
U(0,1)=1 & U(1,1)=1 \\
U(1,0)=1, & h=2,4,6 \ldots \\
U(1, h)=\frac{1}{h !} \quad h=3,5,7 \ldots \\
U(1, h)=0 \quad U(4,1)=\frac{1}{24}, \quad U(5,1)=\frac{1}{120}, \quad U(6,1)=\frac{1}{720} \quad \ldots
\end{array}
$$

Consequently, by substituting the values of $U(k, h)$, we have obtained

$$
U(x, y)=x+y+x y+\frac{1}{2} x^{2} y+\frac{1}{2} x y^{2}+\frac{1}{6} x^{3} y+\frac{1}{24} x^{4} y+\frac{1}{24} x y^{4}+\frac{1}{120} x^{5} y+\frac{1}{720} x^{6} y \ldots
$$

Now the power series $U(x, y)$ can be transformed into multivariate Padé approximation

$m=3, n=2$

$$
p(x, y)=\left|\begin{array}{ccc}
x+y+x y+\frac{1}{2} x^{2} y+\frac{1}{2} x y^{2} & x+y+x y & x+y \\
\frac{1}{6} x^{3} y & \frac{1}{2} x^{2} y+\frac{1}{2} x y^{2} & x y \\
\frac{1}{24} x^{4} y+\frac{1}{24} x y^{4} & \frac{1}{6} x^{3} y & \frac{1}{2} x^{2} y+\frac{1}{2} x y^{2}
\end{array}\right|
$$


$r_{3,2}(x, y)=\left(0,25 x^{2} y^{5}+0,75 x^{3} y^{4}+0,5833333333 x^{4} y^{3}+0,083333 x^{5} y^{2}-0,041666666667 x^{5} y^{3}+0,4166666667 x^{4} y^{4}\right.$

$+0,2916666667 x^{3} y^{5}+0,04166666667 x^{2} y^{6}-0,04166666667 x^{6} y^{2}+0,02083333333 x^{2} y^{7}$

$\left.+0,006944444444 x^{7} y^{2}+0,125 x^{3} y^{6}-0,01388888889 x^{6} y^{3}+0,3541666667 x^{4} y^{5}+0,1875 x^{5} y^{4}\right) \quad /$

$\left(0,25 x^{2} y^{4}+0,08333333333 x^{4} y^{2}+0,5 x^{3} y^{3}+0,04166666667 x^{2} y^{5}-0,04166666667 x^{5} y^{2}\right.$

$\left.-0,08333333333 x^{4} y^{3}-0,02083333333 x^{2} y^{6}+0,006944444444 x^{6} y^{2}-0,02083333333 x^{3} y^{5}-0,02083333333 x^{5} y^{3}\right)$

Table 2: Comparison of the numerical solution of $u(x, y)$ with exact solutions $(y=0.01)$

\begin{tabular}{|c|c|c|c|}
\hline $\boldsymbol{x}$ & $\boldsymbol{u}(\boldsymbol{x}, \boldsymbol{y})$ & $\boldsymbol{r}_{3,2}(\boldsymbol{x}, \boldsymbol{y})$ & $\left|\boldsymbol{u}(\boldsymbol{x}, \boldsymbol{y})-\boldsymbol{r}_{3,2}(\boldsymbol{x}, \boldsymbol{y})\right|$ \\
\hline $\mathbf{0 . 1}$ & 0,1110567092 & 0,1110567093 & 0,0000000001 \\
\hline $\mathbf{0 . 2}$ & 0,2122240276 & 0,2122240282 & 0,0000000006 \\
\hline $\mathbf{0 . 3}$ & 0,3135135881 & 0,3135135784 & 0,0000000097 \\
\hline $\mathbf{0 . 4}$ & 0,4149382470 & 0,4149381645 & 0,0000000825 \\
\hline $\mathbf{0 . 5}$ & 0,5165122127 & 0,5165118515 & 0,0000003612 \\
\hline $\mathbf{0 . 6}$ & 0,6182511880 & 0,6182500305 & 0,0000011575 \\
\hline $\mathbf{0 . 7}$ & 0,7201725271 & 0,7201694638 & 0,0000030633 \\
\hline $\mathbf{0 . 8}$ & 0,8222954093 & 0,8222882864 & 0,0000071229 \\
\hline $\mathbf{0 . 9}$ & 0,9246410311 & 0,9246259708 & 0,0000150603 \\
\hline $\mathbf{1 . 0}$ & 1,027232818 & 1,027203204 & 0,00029614 \\
\hline
\end{tabular}

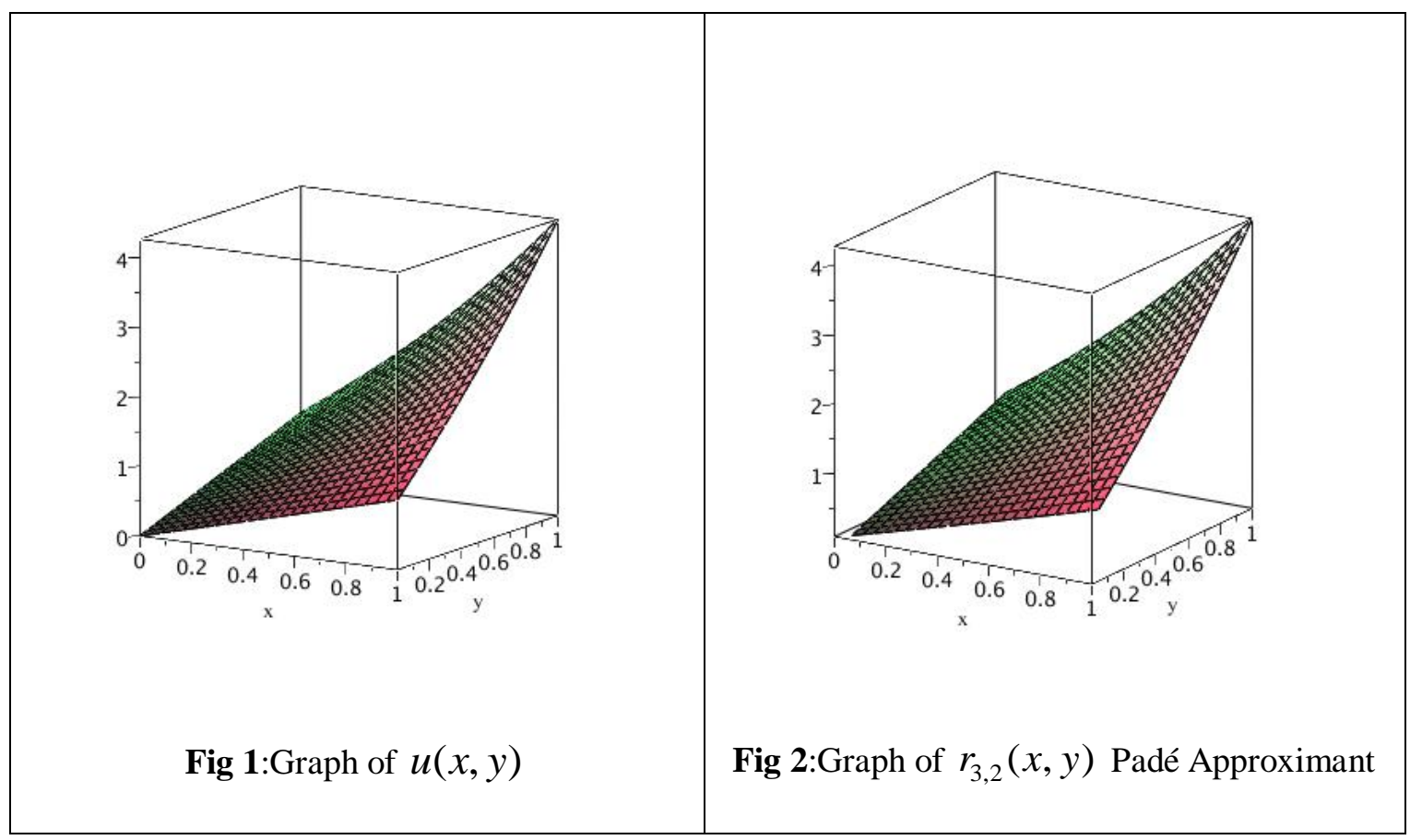


Example 2: Consider the following Helmholtz equation in [13]

$$
\frac{\partial^{2} u(x, y)}{\partial x^{2}}+\frac{\partial^{2} u(x, y)}{\partial y^{2}}+8 u(x, y)=0
$$

with initial conditions

$$
u(0, y)=\sin (2 y), \quad u_{x}(0, y)=0
$$

The exact solution for this equation is

$$
u(x, y)=\cos (2 x) \sin (2 y)
$$

By using the fundamental operations of two-dimensional differential transform method in Table 1, we obtained the following recurrence relation

$$
(k+1)(k+2) U(k+2, h)+(h+1)(h+2) U(k, h+2)+8 U(k, h)=0
$$

By applying the differential transform to initial conditions (26), we have

$$
\begin{gathered}
U(0, h)=0 \quad h \text { is even } \\
U(0,1)=2, \quad U(0,3)=-\frac{4}{3}, \quad U(0,5)=\frac{4}{15}, \quad U(0,7)=-\frac{8}{315} \cdots \\
U(1, h)=0
\end{gathered}
$$

For each $k, h$ substituting Equation (28) into Equation (31) and using recursive method, the values of $U(k, h)$ can be evaluated as follows:

$$
\begin{aligned}
& U(2,1)=-4, \quad U(4,1)=\frac{4}{3}, \quad U(6,1)=-\frac{8}{45}, \quad U(2,5)=-\frac{8}{15} \\
& U(3,4)=\frac{8}{3}, \quad U(3,6)=\frac{16}{135} \quad U(4,5)=\frac{8}{45}, U(6,5)=-\frac{16}{675} \ldots
\end{aligned}
$$

Consequently, by substituting the values of $U(k, h)$, we have obtained

$$
U(x, y)=2 y-4 y x^{2}+\frac{4}{3} y x^{4}-\frac{8}{45} y x^{6}-\frac{4}{3} y^{3}+\frac{8}{3} y^{3} x^{4}+\frac{16}{135} y^{3} x^{6}+\frac{4}{15} y^{5}-\frac{8}{15} y^{5} x^{2} \ldots
$$

Now the power series $U(x, y)$ can be transformed into multivariate Padé approximation

$m=3, n=2$

$$
p(x, y)=\left|\begin{array}{ccc}
2 y-4 y x^{2}-\frac{4}{3} y^{3} & 2 y & 2 y \\
0 & -4 y x^{2}-\frac{4}{3} y^{3} & 0 \\
\frac{4}{3} y x^{4}+\frac{8}{3} y^{3} x^{2}+\frac{4}{15} y^{5} & 0 & -4 y x^{2}-\frac{4}{3} y^{3}
\end{array}\right|
$$

$=32 y^{3} x^{4}+\frac{64}{3} y^{5} x^{2}+\frac{32}{9} y^{7}-\frac{160}{3} y^{3} x^{6}-\frac{352}{9} y^{5} x^{4}-\frac{544}{45} y^{7} x^{2}-\frac{224}{135} y^{9}$ 


$$
q(x, y)=\left|\begin{array}{ccc}
1 & 1 & 1 \\
0 & -4 y x^{2}-\frac{4}{3} y^{3} & 0 \\
\frac{4}{3} y x^{4}+\frac{8}{3} y^{3} x^{2}+\frac{4}{15} y^{5} & 0 & -4 y x^{2}-\frac{4}{3} y^{3}
\end{array}\right|
$$

$r_{3,2}(x, y)=\left(32 y^{3} x^{4}+21.33333333 y^{5} x^{2}+3.555555556 y^{7}-53.33333333 y^{3} x^{6}-39,11111111 y^{5} x^{4}-12.08888889 y^{7} x^{2}-1.659259259 y^{9}\right) /$ $\left(16 y^{2} x^{4}+10.66666667 y^{4} x^{2}+1.777777778 y^{6}+5.333333333 y^{2} x^{6}+12.44444444 y^{4} x^{4}+4.622222222 y^{6} x^{2}+3.555555556 y^{8}\right)$

Table 3. Comparison of the numerical solution of $u(x, y)$ with exact solutions $(y=0.01)$

\begin{tabular}{|c|c|c|c|}
\hline $\boldsymbol{x}$ & $\boldsymbol{u}(\boldsymbol{x}, \boldsymbol{y})$ & $\boldsymbol{r}_{3,2}(\boldsymbol{x}, \boldsymbol{y})$ & $\left|\boldsymbol{u}(\boldsymbol{x}, \boldsymbol{y})-\boldsymbol{r}_{3,2}(\boldsymbol{x}, \boldsymbol{y})\right|$ \\
\hline $\mathbf{0 . 1}$ & 0,01960002483 & 0,01960002211 & 0,0000000027 \\
\hline $\mathbf{0 . 2}$ & 0,01841999150 & 0,01841982343 & 0,0000001680 \\
\hline $\mathbf{0 . 3}$ & 0,01650560359 & 0,01650374840 & 0,0000018551 \\
\hline $\mathbf{0 . 4}$ & 0,01393312265 & 0,01392310528 & 0,0000100173 \\
\hline $\mathbf{0 . 5}$ & 0,01080483520 & 0,01076847338 & 0,0000363618 \\
\hline $\mathbf{0 . 6}$ & 0,00724457301 & 0,00714230441 & 0,0001022685 \\
\hline $\mathbf{0 . 7}$ & 0,00339195342 & 0,00315152090 & 0,0002404325 \\
\hline $\mathbf{0 . 8}$ & $-0,00060466011$ & 0,00010990339 & 0,0004943738 \\
\hline $\mathbf{0 . 9}$ & $-0,00459647754$ & $-0,00551174491$ & 0,0009152673 \\
\hline $\mathbf{1 . 0}$ & $-0,00844388148$ & $-0,00999975003$ & 0,0015558685 \\
\hline
\end{tabular}

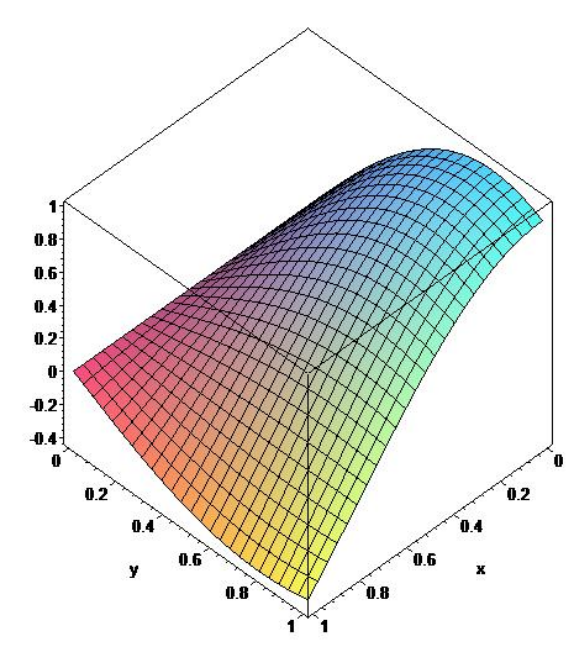

Fig 3: Graph of $u(x, y)$

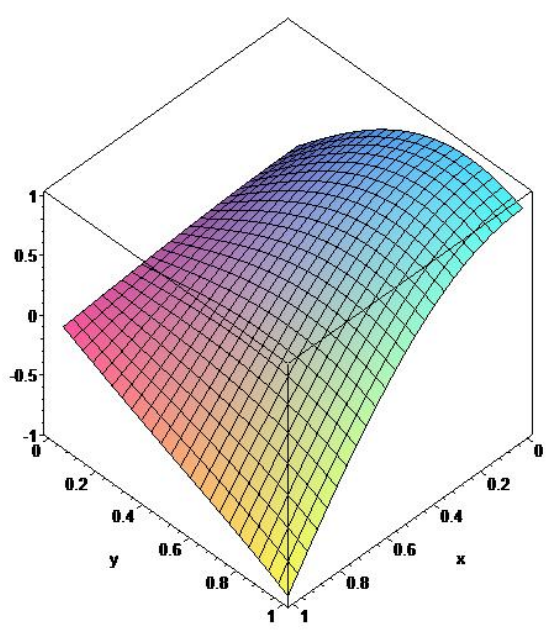

Fig 4: Graph of $r_{3,2}(x, y)$ Padé Approximant

\section{CONCLUSION}


In this study, we have introduced Differential Transform Method and Padé Approximation to solve Helmholtz Differential Equations. The numerical results of the examples are in good-agreement with the exact solutions. It may be concluded that the method is an efficient way to find numerical solutions for initial value problems. On the other hand, the results are quite reliable. Therefore, this method can be applied to many partial differential equations.

\section{REFERENCES}

[1] Biazar, J., Eslami, M., Differential Transform method for Solving Helmholtz Equation, World Applied Sciences Journal 10 (2): 166-168 (2010)

[2] Burden, R. L., Faires, J. D., Numerical Analysis, PWS Publishing Company, Boston (1993)

[3] Gerald, C. F., Wheatley, P. O., Applied Numerical Analysis, Addison Wesley, California (1994)

[4] Cattani, C and Rushchitsky, J.J. Solitary elastic waves and elastic wavelets, Int. Appl. Mech., 39, No. 6, 741-752 (2003).

[5] Nabavi, M., Siddiqui, M. H. K. and Dargahi, J., A new 9point sixth-order accurate compact finite-difference method for the Helmholtz equation, Journal of Sound and Vibration, vol. 307, no. 3-5, pp. 972-982, (2007).

[6] El-Sayed, S. M., Kaya, D., Comparing Numerical Methods for Helmholtz Equation Model Problem, Applied Mathematics and Computation 150: 763-773 (2004)

[7] Zhou, J. K., Differential Transform and its Applications for Electrical Circuits (Wuhan: Huarjung University Press) (1986)

[8] Ayaz, F. On the Two-Dimensional Differential Transform Method, Applied Mathematics and Computation, 143:361374 (2003)

[9] Kurulay M., Bayram M., Solving of Nonlinear TimeFractional Schrodinger Equation by Two-Dimensional Differential Transform Method, World Applied Sciences Journal, (2010)

[10] Turut, V., Çelik, E., Yiğider, M., Multivariate Padé Approximation for Solving Partial Differential Equations (PDE), International Journal for Numerical Methods in Fluids, 66 (9), 1159-1173 (2010)

[11] Cuyt, A., Wuytack, L., Nonlinear Methods in Numerical Analysis, Amsterdam (1987)

[12] Yiğider, M., Çelik, E., The numerical solution of partial differential-algebraic equations, Advances in Difference Equations, 2013:8 (2013)

[13] Noor, M. A., Mohyud-Din, S. T., Modified variational iteration method for solving Helmholtz equations, Computational Mathematics and Modeling, Vol. 20, No. 1, pp: 40-50 (2009) 